\title{
Application of ANSYS APDL in the Design of Piezoelectric Transducer
}

\author{
Fuxu Li ${ }^{1, a}$, Guangji Li ${ }^{2, b^{*}}$ \\ 1,2100 Haiquan Road, Fengxian District, Shanghai, China \\ alifuxu@163.com, ${ }^{\mathrm{b}}$ liguangji@126.com
}

\begin{abstract}
Keywords: piezoelectric transducer; resonance frequency; vibration mode; coupling; parametric design language.

Abstract. Piezoelectric transducer is more and more widely used, how to obtain its resonant frequency and vibration mode according to the material parameters is the main problem to solve in the design process. Due to the coupling in working process, the vibration theory of the traditional plate has been unable to solve these problems. In this paper, with the help of finite element software ansys 15.0, using parametric design language, by establishing a finite element model and applying specific boundary conditions and solution, the final simulation results are close to the test value of the actual sample, fully demonstrated the validity of ANSYS15.0 in solving such problems.
\end{abstract}

\section{Introduction}

In addition to the extensive application in the daily life, the ultrasonic atomizer is also used in the medical atomizing inhalation therapy. Ultrasonic atomizer use electronic high-frequency oscillation by ceramic atomization of high frequency resonance, molecular structure of liquid water will break up and produce water mist[1]. As the core of ultrasonic atomization piezoelectric transducer, it is composed of a piezoelectric ceramic and metal cover plate, its principle is to make use of the converse piezoelectric effect of piezoelectric ceramic, and converts electrical energy into mechanical energy. When applying an electric field, piezoelectricity slice deformation due to the inverse piezoelectric effect, and the deformation is equal to the frequency of the alternating electric field, so that the piezoelectric transducer can generate an ultrasonic wave[2].

How to obtain the atomization piece of work frequency and vibrational state is the major problem in the design and optimization process. The traditional method is to set up the free vibration equation of the piezoelectric plate, and use the analytic method to solve it. However, this method is too complicated, and the results are not intuitive[3].Due to the piezoelectric coupling effect, it is very difficult to carry on the theoretical analysis of the piezoelectric energy transfer film from the perspective of the mechanical and electrical coupling, and most of the work is carried out in special circumstances, and it is difficult to get the explicit solution for general situation. Because of the complex boundary condition and stress state of the piezoelectric ceramic plate, the research on the traditional method is not only time-consuming, but also has a strong limitation[4]. With the development of the computer simulation technology, the use of the finite element method can break the above restrictions. In this paper, with the aid of finite element software ansys15.0, using parametric design language, by establishing a finite element model and applying specific boundary conditions and solution, the final simulation results are close to the test value of the actual sample, fully demonstrated the validity of ANSYS15.0 in solving such problems.

\section{Theoretical basis}

The introduction of ANSYS APDL. ANSYS software is a large general-purpose CAE software which is widely used in many industrial fields. It provides a variety of physical field analysis, set structure, thermal, electromagnetic, body, acoustic and other physical field solving function in one, but also to achieve a multi physical field coupling analysis[5]. The coupled field analysis is an analysis of the interaction between two or more engineering physical fields. The piezoelectric analysis is to consider the interaction between the structure and the electric field, and to solve the distortion caused by the 
applied voltage. The piezoelectric analysis is one of the direct coupled field analysis, and its type can be steady, mode, pre-stress mode, harmonic, pre-stress harmonic and transient analysis[6]. Like all of the analysis of finite element software, ANSYS software analysis process include three steps: establishing analysis model and adding boundary condition, solving calculation and results analysis. ANSYS software provides the function of the analysis of the command flow, which is the parametric design language. It can use the first analysis of the log files, the user can complete as many analysis by means of modify, thus greatly reducing the modified model to analyze the time required[7].

Piezoelectric equation. The piezoelectric properties of piezoelectric materials relate to the relationship between mechanical and electrical. The piezoelectric equation is an expression that describes the relationship between the mechanical and electrical quantities of piezoelectric materials. [8] The second kinds of piezoelectric equations are used in the ANSYS of the piezoelectric coupling problem. In linear piezoelectricity the equations of elasticity are coupled to the charge equation of electrostatics by means of piezoelectric constants:

$$
\begin{aligned}
& \{T\}=\left[c^{E}\right]\{S\}-[e]\{E\} \\
& \{D\}=[e]^{T}\{S\}+\left[\varepsilon^{S}\right]\{E\}
\end{aligned}
$$

where:

$\{T\}=$ stress vector, $\{D\}=$ electric flux density vector, $\{S\}=$ elastic strain vector, $\{E\}=$ electric field intensity vector, $\left[c^{E}\right]=$ elasticity matrix, $[e]=$ piezoelectric stress matrix, $\left[\varepsilon^{S}\right]=$ dielectric matrix

Basic principle of modal analysis. Modal analysis is used to analyze the natural frequency and mode shape of the structure, which is the natural frequency and vibration mode of the structure, Natural frequency and vibration mode is an important characteristic parameters of piezoelectric transducer, which determines whether the piezoelectric transducer can achieve the best power conversion and transmission[9]. If the continuum is considered as a multi freedom system, the matrix representation of the continuum dynamics model is:

$$
[M]\{[C]\{u+[K]\{u\}=\{F\}
$$

Where:

$[M]=$ Mass matrix, $[C]==$ Damping matrix, $[K]=$ Stiffness matrix, $\{F\}=$ External excitation force vector, $\{=$ Node acceleration vector, $\{\iota=$ Node velocity vector, $\{u\}=$ Node displacement vector

When $\{F\}=\{0\}$, the corresponding analysis is the modal analysis[10]:

$$
[M]\{+[C]\{+[K]\{u\}=\{0\}
$$

The problem becomes the eigenvalue problem for finding the solution of 3 nonzero values. 


\section{Case simulation}
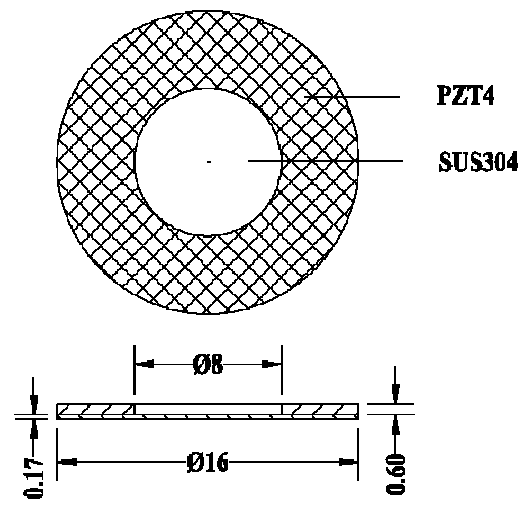

Figure 1 Piezoelectric plate structure diagram

Problem Description. The analysis object is commonly used in the market for the atomization of the piezoelectric plate, which is composed of a piezoelectric ceramic ring and a metal plate. The properties of ceramic materials is in table 1 and structure as shown in figure 1.

Table 1 Piezoelectric material parameters.

\begin{tabular}{cccc}
\hline Material & Young's modulus $/ \mathrm{GPa}$ & Density $/ \mathrm{kg} \cdot \mathrm{m}^{-3}$ & Poisson's ratio \\
\hline SUS304 & 206 & 7930 & 0.3 \\
PZT-4 & & 7500 & \\
\hline
\end{tabular}

Dielectric constants matrix: $[\varepsilon]=\left[\begin{array}{lll}6.45 & & \\ & 6.45 & \\ & & 5.61\end{array}\right] \times 10^{-9} \mathrm{C}\left[\mathrm{m}^{-1}\right.$

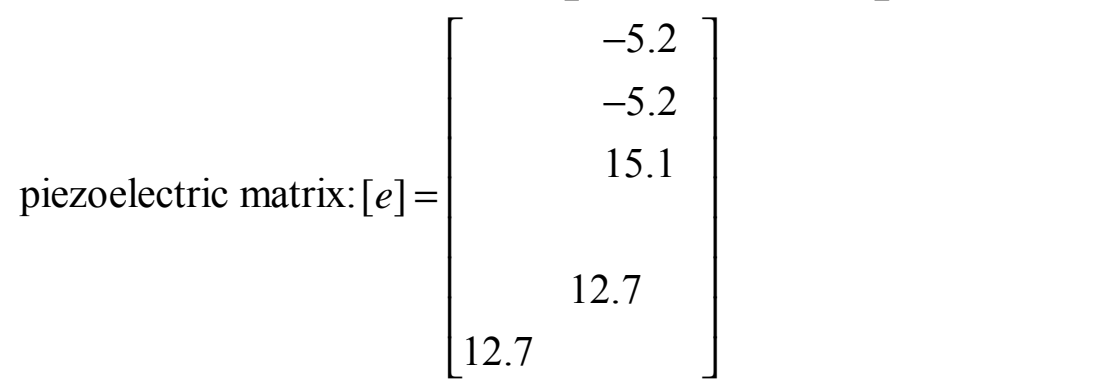

Elastic Coefficient Matrix: $[c]=\left[\begin{array}{lllllll}13.9 & 7.78 & 7.43 & & & \\ 7.78 & 13.9 & 7.43 & & & \\ 7.43 & 7.43 & 11.5 & & & \\ & & & 3.06 & & \\ & & & & 3.06 & \\ & & & & & 3.06\end{array}\right]$

\section{Analysis code.}

\section{/PREP7}

/TITLLE,PZT4

ET,1,SOLID5

ET,2,SOLID45

MP,DENS, 1,7500

MP,PERX,1,6.45E-9 ! Dielectric constants

MP,PERY,1,6.45E-9

MP,PERZ,1,5.61E-9

TB,PIEZ, 1

TBDATA, 3,-5.2

TBDATA, $6,-5.2$
! Define the model parameters

! Piezoelectric 8-node brick

! Solid structure unit 8-nodes brick 


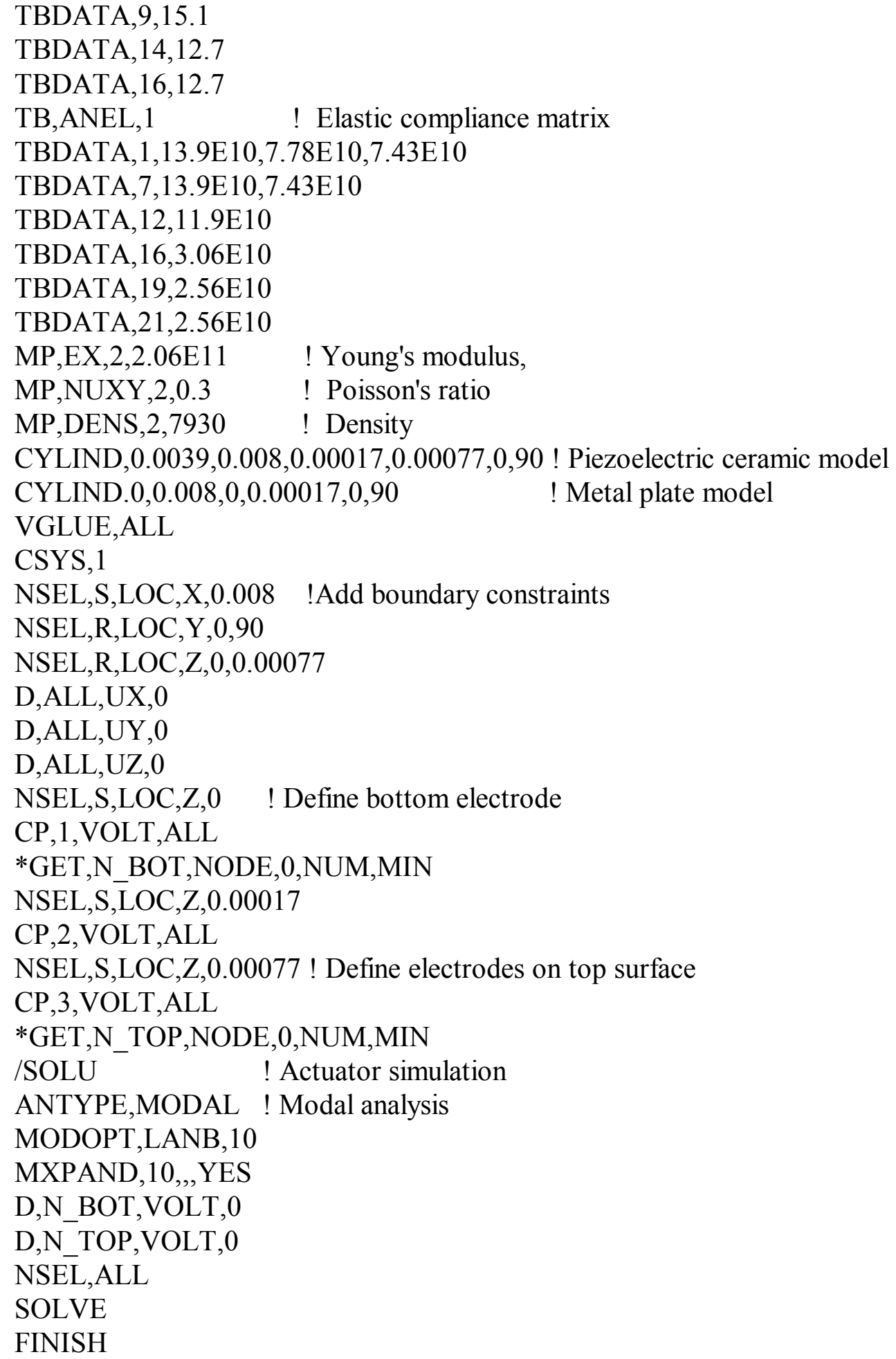

\section{Results view}

Using general (POST1) processor view micro atomizer plate vibration, the first ten order vibration mode and frequency is obtained, according to the vibration mode choice of longitudinal vibration results such as Figure 2 and figure 3 and Figure 4 and Figure 5. 


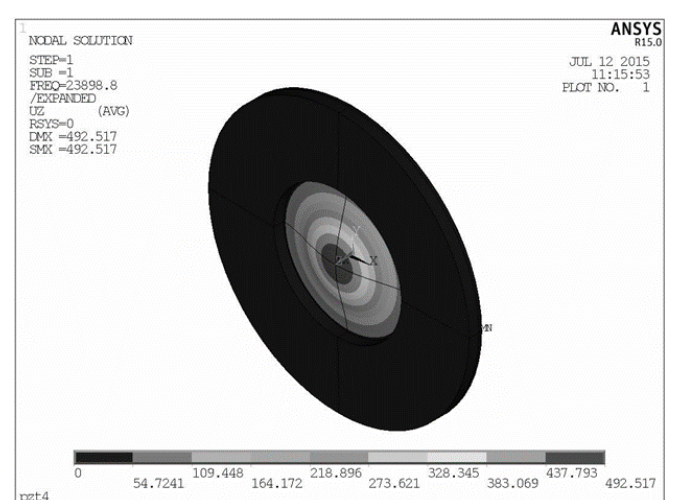

Fig. 2 The first order vibration cloud

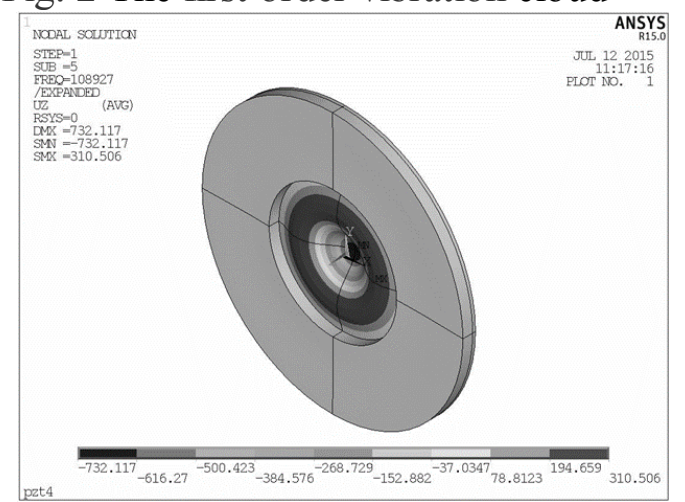

Fig. 4 The fifth order vibration cloud

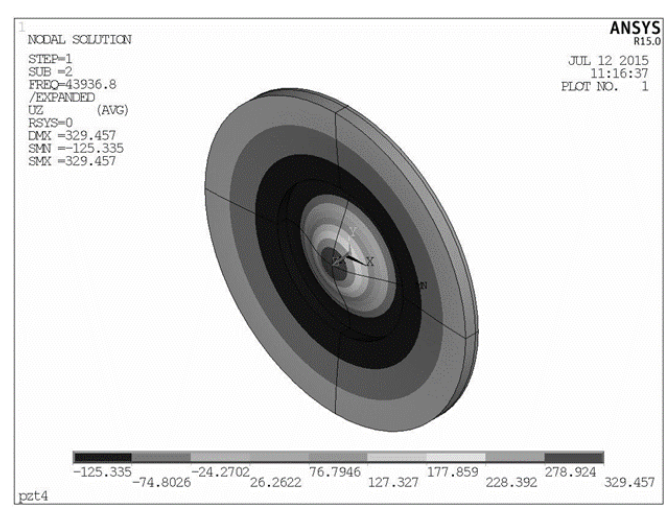

Fig 3 The second order vibration cloud

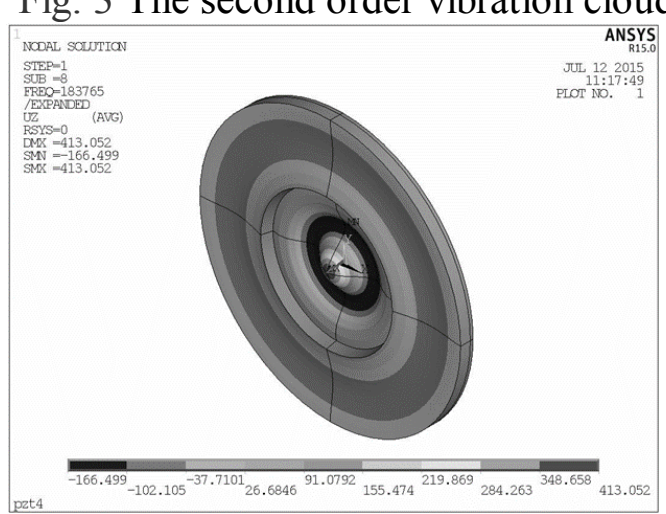

Fig. 5 The eighth order vibration cloud

From figure 4 to figure 7 shows the first order and second order, fifth, eighth order vibration mode is thickness direction vibration, and it can produce large center displacement, the droplets in these conditions are stable and fast, And the maximum deformation of the fifth stage can be used as the working frequency of the piezoelectric plate, Through digital bridge with TH2818 test sample frequency is $109164 \mathrm{~Hz}$. The table 2 is comparison of frequency results

Table 2 Comparison of frequency results

\begin{tabular}{cccc}
\hline Model & Theoretical value $/ \mathrm{HZ}$ & Test value/HZ & Simulation value/HZ \\
\hline HA-16 & 109000 & 109164 & 108927 \\
\hline
\end{tabular}

\section{Conclusion}

The above simulation results show that:

(1)It can be found that the simulation value and the actual value and the theoretical value are very close through simulation analysis and actual sample testing. The validity of the ANSYS software in the analysis of the piezoelectric coupling is proved.

(2)The vibration modes and vibration modes of each order can be observed by the post processor, and it can choose the operating frequency according to the actual situation, which provides a more intuitive results for the design and optimization of the piezoelectric transducer.

(3) Compared with the traditional method of vibration mode analysis, using the ANSYS parametric design language in the software of piezoelectric coupling analysis more quickly, and it can simplify the problem analysis process and save the design time.

(4)It can simplify the modeling and optimization process by using ANSYS software internal material containing relevant piezoelectric materials. And it provides an effective support for further study on the ultrasonic atomizer design optimization.

\section{Acknowledgements}

This paper is supported by the National Natural Science Fund Project (Project number is 51205257). 


\section{References}

[1] H.Y. Zhao, L.C. Chai. The analysis of ultrasonic atomizer droplets flying time, J. Journal of Sichuan Institute of Technology. 23 (2010) 88-90. (In Chinese)

[2] C. Cheng, F. Zhu, B. Fu. Modeling and Analysis of Sandwich Piezoelectric Ultrasonic Transducer Based on ANSYS, J. Mechanical Engineer, 1 (2015) 125-127. (In Chinese)

[3] S. Chen, J. Yang. Analysis of Radial Vibration Modes of Round Piezoelectric Ceramics Based on ANSYS, J. Computer Simulation, 3 (2009) 318-321. (In Chinese)

[4] D.Y. Jiang, Z.Y. Zheng, L. Li, S. Ren. The Analyses of the Vibration Model of Piezoelectric Ceramic Piece Based on ANSYS, J. Journal of Sensing Technology, 16 (2004) 452-456. (In Chinese)

[5] C.X. Zeng, L. Jiang. The Finite Element Analysis of Piezoelectric Transducer Based on ANSYS, J. Instrument Technology, 6 (2011) 31-33. (In Chinese)

[6] ANSYS, Inc. ANSYS Couple Field Analysis Guide, Release (1999) 36-3.

[7] G.D. Luan, J.D. Zhang, R.Q. Wang. Piezoelectric Transducer and Transducer Array. Science Press, Beijing, 2005. (In Chinese)

[8] S.Y. Lin. The Principle and Design of Ultrasonic Transducer. Peking University Press,Beijing, 2004.(In Chinese)

[9] J.B. Lin, Y.X. Chen. Ansys14 Complete Self-study Manual, Electronics Industry Press, Beijing, 2013. (In Chinese)

[10]J.Q. Liu, J.C. Yan. Modal analysis of ultrasonic knife cutting system, J, Journal of Harbin Institute of Technology. 33 (2001) 435-438. (In Chinese) 